\title{
Effect of Omega-3 Fatty Acids on Hormonal Profile and Ovarian Stromal Blood Flow in Patients with Polycystic Ovary Syndrome
}

\author{
Original \\ Article \\ Taiseer Maarouf, Doaa M. Saleh, Aalaa A. Tantawy, Passant M. Eid \\ Department of Obstetrics \& Gynecology, Faculty of Medicine, Al-Azhar University for Girls, \\ Egypt
}

\begin{abstract}
Background: In Polycystic ovary syndrome (PCOS) life style and dietary changes are recommended as the first line of management; however, the optimal nutritional management is not certain yet. Polyunsaturated fatty acids (PUFAs), particularly long-chain (omega-3) PUFAs, is known to improve overall human health and its widely used in managing infertility, but its role in PCOS management is still uncertain.

Objective: The purpose of this study was to assess if omega-3 fatty acids supplementation for Polycystic ovary syndrome patients will have a positive effect on body weight, Hirsutism score, Menstrual cyclicity, hormonal status and Doppler flow to the Uterine and Ovarian stromal arteries.

Study Design: A randomized prospective trial.

Patients and Methods: A total of 67 patients with Polycystic ovary syndrome were randomized into two groups. Group I patients who received omega-3 fatty acid 1 gram daily capsules and group II patients who received no treatment for 3 months. Changes in weight, BMI, menstrual cycle length, modified Ferriman Gallway score, serum FSH, LH ,free Testosterone levels and Doppler of ovarian stromal and uterine arteries between baseline and the 3 months study period were compared between the 2 groups.

Results: After 3 months supplementation with omega-3 fatty acid capsules, there was a statistically significant decrease in menstrual cycle length in the study group in comparison to the control group. Also, there was a statistically significant decrease in the pulsatility index (PI) of the uterine artery Doppler in the study group, but there was no change in the weight, BMI, ovarian stromal blood flow. Also, measures of FSH, LH and free testosterone did not show statistically significant changes for either group.

Conclusion: Omega-3 fatty acids supplementation is effective in improving menstrual cyclicity and uterine artery blood flow in patients with PCOS and may be an helpful option for some patients with PCOS especially those who have defective endometrial receptivity and subsequent infertility or repeated miscarriages because of increased uterine artery resistance.
\end{abstract}

Key Words: Omega-3 fatty acid, ovarian stromal blood flow, PCOS, uterine artery Doppler

Received: $21^{\text {st }}$ July 2019, Accepted: $8^{\text {th }}$ August 2019

Corresponding Author: Doaa M. Saleh, Department of Obstetrics \& Gynaecology, Faculty of Medicine, Al Azhar University for Girls, Madinet Nasr, Abbassia, Cairo, Egypt, Tel.: +20 1112987286, E-mail: dr.dodogyne@gmail.com

ISSN: 2090-7625, November 2019, Vol.9, No. 4

\section{INTRODUCTION}

Polycystic ovary syndrome (PCOS) is the most common endocrinopathy in women of reproductive age. Worldwide prevalence of PCOS estimates vary widely from $8-13 \%$ of reproductive-aged women with up to $70 \%$ of affected women remaining undiagnosed. ${ }^{[1]}$

It is one of the most complicated human disorders, without its proper diagnosis and treatment it can lead to serious medical problems as diabetes mellitus, hypertension, cardiovascular diseases, depression and endometrial carcinoma ${ }^{[2]}$.

The main complaints of PCOS patients is hyperandrogenism. causing Hirsutism, menstrual irregularities and infertility ${ }^{[3]}$.
PCOS patients have increased impedance of the uterine reducing uterine perfusion, and that interferes with implantation and increases the possibility of spontaneous abortion $^{[4]}$.

There is high blood flow in the ovarian stroma of PCO patients. Lower PI and RI were seen in PCOS ovaries in comparison to normal ovaries. This increased stromal perfusion may explain why PCOS patients have exaggerated ovarian response to controlled ovarian stimulation and are at more risk of having ovarian hyperstimulation syndrome ${ }^{[5]}$.

In management of PCOS women, especially for PCOS patients with obesity, lifestyle and nutrition interventions and weight loss are the first step in the recommendations of treatment in the guidelines ${ }^{[6]}$. 
Among dietary management for PCOS, omega- 3 fatty acids play an important role in weight loss, improving insulin sensitivity and ovulation enhancement. ${ }^{[5]}$

The only essential types of all dietary fatty acids are omega-3 and 6 polyunsaturated fatty acids; because all other fatty acids can be synthesized from the other shorter chain fatty acids in the human body. ${ }^{[6]}$

Polyunsaturated fatty acids (PUFAs) have 16 to 22 carbon atoms and more than one double bond. The longchain omega-3 Poly unsaturated fatty acids; namely, eicosapentaenoic acid (EPA) and docosahexaenoic acid (DHA) have many crucial effects in human health. ${ }^{[7]}$

Over the past 20 years, many human trials and experimental studies have confirmed the hypotriglyceridemic, anti-inflammatory and antithrombotic benefits of omega-3 fatty acids ${ }^{[6]}$. Omega-3 PUFAs have a broad range of actions in reproductive tissues including affecting membrane fluidity, intracellular cell-signaling cascades and susceptibility to oxidative injury ${ }^{[5]}$ as well as decreases insulin resistance, inflammation and obesity, lower plasma cholesterol level which may lead to reduced steroid hormone synthesis. ${ }^{[8]}$

Omega-3 PUFAs are direct precursors of prostaglandin synthesis which participate in the regulation of reproductive endocrine hormones ${ }^{[9]}$. A number of clinical trials studied the effect of omega-3 fatty acids in polycystic ovary syndrome patients but they show controversial results ${ }^{[9]}$.

\section{AIM OF THE WORK}

The aim of this study was to discover if omega-3 PUFA supplementation would show beneficial effects on body weight, hormonal levels and Doppler of the uterine and ovarian vessels in women with polycystic ovary syndrome.

\section{PATIENTS AND METHODS}

Medical ethical committee at Al-Azhar University Faculty of Medicine for Girls approved the study design. It was done during the period from January 2016 to December 2018.

Eighty women complaining of irregular menstrual cycles and or/hirsutism were recruited from the General Gynecology or Infertility Clinics in Al-Zahraa University Hospital.

Sample Size : Calculation was done using the comparison of serum free testosterone before and after giving omega-3 in cases with PCOS, as it was the primary outcome of our study. As reported in previous publications, the mean $\pm \mathrm{SD}$ of pre-treatment serum free testosterone in PCO cases was approximately $1.4 \pm 0.9 \mathrm{ng} / \mathrm{ml}$, while it was $0.85 \pm 1.37 \mathrm{ng} / \mathrm{ml}$ after treatment. Accordingly, we calculated that the minimum proper sample size was 37 patients who were able to detect a real difference of $0.55 \mathrm{ng} / \mathrm{ml}$ with $80 \%$ power at $=0.05$ level using Paired t-test. Sample size calculation was done using Stats Direct statistical software version 2.7.2 for MS Windows, StatsDirect Ltd., Cheshire, UK.

Inclusion criteria : Patients in reproductive age group 18 -39 years, BMI ranging from 20 to 35, diagnosed as PCOS according to the revised Rotterdam criteria 2004 with 2 out of the 3 following criteria being diagnostic of the condition: Oligo-ovulation and/or anovulation, clinical and/or biochemical signs of hyperandrogenism hirsutism or hyperandrogenemia and/or (PCOM) diagnosed by ultrasonography (Increase ovarian volume $(>10 \mathrm{cc})$ or follicles number $=12$ or more, measuring 2 to $9 \mathrm{~mm}$ ).

Exclusion criteria : Secondary causes of hyperandrogenism were excluded by history examination and laboratory tests, as late onset congenital adrenal hyperplasia, hyperprolactinemia, adrenal/ovarian tumors, Cushing syndrome and thyroid disorders. Taking nutritional supplements or any medications was likely to influence the results including hormonal contraception, antihypertensives, antiplatelets and anti-inflammatory agents, and patients with systemic illnesses, such as renal, hepatic, hyperlipidemias, hypertension, diabetes, cardiovascular disorders and presence of local uterine or adnexal diseases or history of tubal or ovarian surgery or pathology.

\section{Methods:}

1. Explanation of the study to all the participated patients and taking verbal consent from them.

2. Detailed history from each patient including age, parity, years since married, menstrual and obstetric histories and medical history to exclude any medical disorders.

3. General, abdominal and pelvic examination, height and weight were determined for all patients and BMI was calculated $(\mathrm{BMI}=$ Weight $(\mathrm{KG}) /$ Height $(\mathrm{M} 2))$

4. Hormonal assay: serum FSH, LH, free testosterone, prolactin, $17 \mathrm{OH}$ Progesterone, TSH and DHEA-S level serum samples.

5. Transvaginal ultrasound (Two-dimensional sonographic and color Doppler examinations) was performed using 6.5-megahertez transvaginal probe Samsung co. Model HS 70. The patients were asked to evacuate bladder before examination and examination was done in supine position with knee flexed and lower limb abducted. After scanning uterine position, size, endometrial thickness and uterine morphology, localization of the ovaries is on each side of the uterus, close to the lateral wall of the pelvis and their size, shape, volume and follicle count were measured. Volumes were calculated by measuring the maximum longitudinal, anteroposterior and transverse diameters and using the formula based on 
ellipsoid: (Volume $=0.523 \mathrm{abc}$ ) ; $\mathrm{a}$ is the length, $\mathrm{b}$ is the width and $\mathrm{c}$ is the thickness of the ovary.

Both uterine arteries were identified as they turned cranially to make their ascent to the uterine body and located lateral to the cervix by color Doppler imaging. The pulsed Doppler gate placed over the artery and the resulting waveform obtained from the pulsed Doppler analyzed by resistance (RI) and pulsatility indices (PI). Both resistive and pulsatility indices were calculated by the machine.

Then, color Doppler imaging was used to assess the vascularization of the ovarian stroma. The gate of the Doppler was positioned on the small vessels in the ovarian stroma, while the vessels close to the surface and the capsule of the ovary were not measured.

Randomization : The study included 67 patients who were randomized into 2 groups (34 study group and 33 control group) according to computer generated randomization.

Group 1 (study group) included 34 patients who received 1 gram of omega-3 PUFA in the form of (omega-3 plus capsule) (Sedico Company, Egypt) once daily for 12 weeks; Group 2 (control group) included 33 patients who received no treatment.

Primary outcome : The effect of omega-3 fatty acid on hormonal profile (FSH, $\mathrm{LH}$ and free testosterone) were assessed after 12 weeks.

Secondary outcome : Effects on body weight, BMI, menstrual cycle length, ovarian stromal blood flow and uterine artery blood flow by Doppler ultrasound were assessed after 12 weeks.

Statistical methods : Data were described as mean \pm standard deviation (SD), comparison of the numbers between, before and after the trial in the study and control groups was done using student T-test for independent samples in comparing 2 groups when normally distributed and Mann Whitney $U$ test for independent samples when not normally distributed. A (P value) probability value less than 0.05 is considered statistically significant. Statistical computer calculations were done using Microsoft Excel 2016.

\section{RESULTS}

This figure shows the study flowchart. Eighty patients were screened for eligibility to join the study, in which nine patients were not eligible (two patients consumed omega-3 before the study, one patient had hyperprolactinemia, one patient had high DHEA-S and five patients had hypothyroidism) four patients did not desire to participate and refused informed consent as they wanted to receive infertility treatment in the next 3 months.

Sixty-seven eligible patients were randomly divided to omega-3 or control groups and were followed for 3 months. During follow-up, one patient got pregnant, one patient undergone laparoscopic ovarian drilling and two patients were lost to follow up. Finally, 32 patients in omega- 3 group and 30 patients in control groups completed the study and were analyzed.

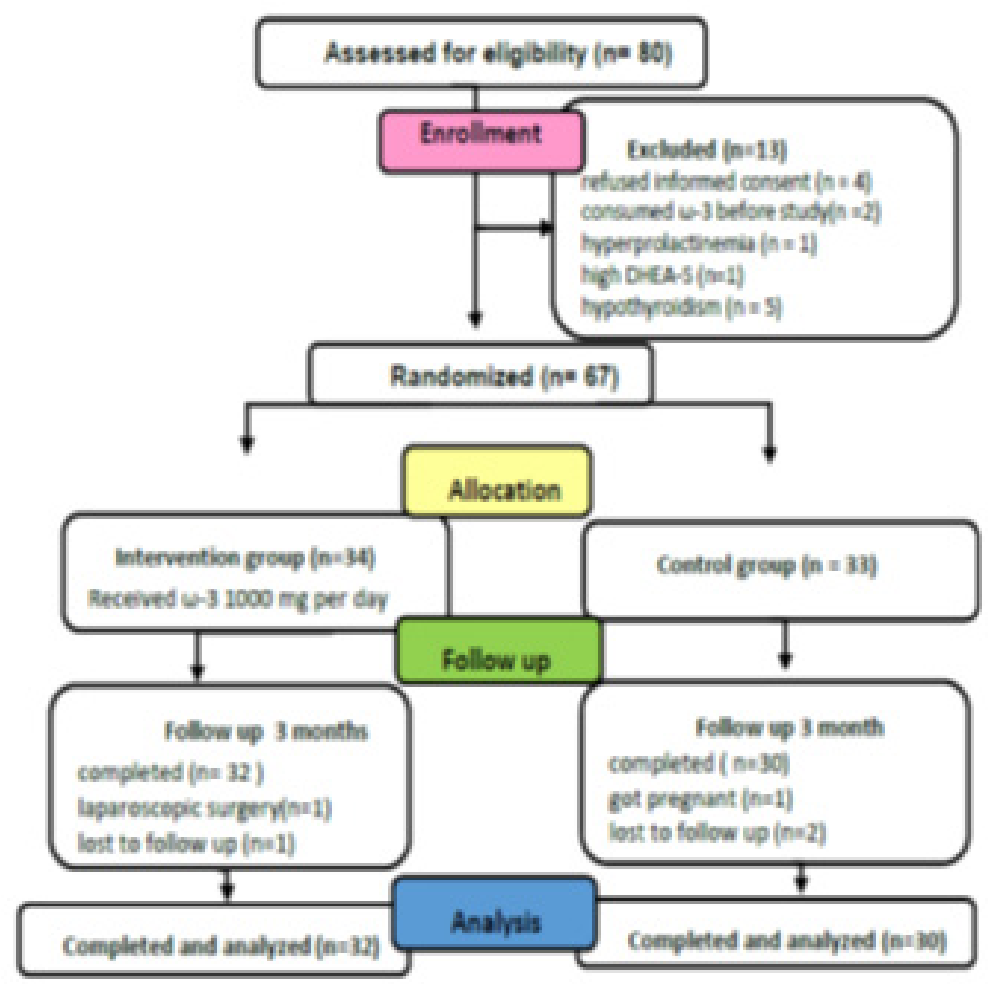


Table 1 shows patients data and basal endocrine and sonographic studies in the intervention and control group. All variables between groups were similar and no significant differences were found between the two groups before the study $(P>0.05)$

Table 1 : Background characteristics of participants

\begin{tabular}{|c|c|c|c|}
\hline Variable & Study group & $\begin{array}{l}\text { Control } \\
\text { group }\end{array}$ & P-value \\
\hline Age (year) & $25.7 \pm 0.5$ & $25.1 \pm 0.6$ & 0.42 \\
\hline Parity & $1(0-2)$ & $1(0-2)$ & $0.91 *$ \\
\hline BMI (kg/m2) & $27.3 \pm 0.63$ & $28.4 \pm 0.6$ & 0.24 \\
\hline F G score & $12.8 \pm 3.4$ & $13.7 \pm 3.3$ & 0.33 \\
\hline $\begin{array}{l}\text { Menstrual } \\
\text { cycle }\end{array}$ & $47 \pm 8.99$ & $47 \pm 8.8$ & 0.33 \\
\hline FSH (IU) & $4.5 \pm 1.6$ & $4.4 \pm 2.0$ & $0.39 *$ \\
\hline LH (IU) & $9.4 \pm 5.4$ & $8.2 \pm 4.4$ & $0.51 *$ \\
\hline LH/FSH ratio & $2.18 \pm 1.15$ & $2.23 \pm 1.36$ & $0.79 *$ \\
\hline $\begin{array}{l}\text { Free T } \\
(\mathrm{pg} / \mathrm{ml})\end{array}$ & $1.5 \pm 0.67$ & $1.6 \pm 0.7$ & $0.47^{*}$ \\
\hline $\mathrm{E} 2(\mathrm{pg} / \mathrm{ml})$ & $169 \pm 67$ & $199 \pm 76$ & $0.29 *$ \\
\hline PRL( ng/ml) & $8.8 \pm 9.1$ & $9.6 \pm 5.6$ & $0.69 *$ \\
\hline $\begin{array}{l}\text { 17-OHP } \\
(\mathrm{ng} / \mathrm{ml})\end{array}$ & $2.1 \pm 1.1$ & $2.3 \pm 1.2$ & $0.67^{*}$ \\
\hline $\begin{array}{l}\text { TSH } \\
(\mathrm{mIU} / \mathrm{mL})\end{array}$ & $2.6 \pm 1.27$ & $2.21 \pm 1.3$ & $0.19^{*}$ \\
\hline $\begin{array}{l}\text { DHEA- } \\
\text { S(Ug/dl) }\end{array}$ & $301 \pm 157$ & $244 \pm 107$ & $0.09^{*}$ \\
\hline $\begin{array}{l}\text { Mean ovarian } \\
\text { volume }\end{array}$ & $11.01 \pm 1.5$ & $11.14 \pm 2.18$ & 0.36 \\
\hline $\begin{array}{l}\text { Antral } \\
\text { follicle count }\end{array}$ & $16.4 \pm 3.9$ & $15.2 \pm 3.6$ & 0.19 \\
\hline
\end{tabular}

Data expressed as mean $\pm \mathrm{SD}$ or median (range), $P$-values calculated by Independent sample $\mathrm{t}$ test or *Mann-Whitney U test.

SD: Standard deviation. F G score Ferriman Gallway score ,FSH: follicle stimulating hormone; $\mathrm{LH}$ : luteinizing hormone; free T: free testosterone; E2: estradiol; PRL: prolactin; 17-OHP: $17 \alpha$ - hydroxyprogesterone; DHEAS dehydroepiandrosterone sulfate; TSH: thyroid stimulating hormone.

In table 2 , the comparison of weight, BMI, modified Ferriamn Gallway score between omega-3 group and control groups before and after treatment showed no statistically significant change between the 2 groups before and after the study. $(P>0.05)$.

Interval between periods become much shorter in omega-3 group compared to control group $(28.83 \pm 4.68$ vs. $47.11 \pm 8.72$ days, respectively, $P<0.004)$.

Table 2 : Comparison before and after treatment

\begin{tabular}{|c|c|c|c|}
\hline Variable & Study group & $\begin{array}{l}\text { Control } \\
\text { group }\end{array}$ & P-value \\
\hline \multicolumn{4}{|c|}{ Weight (KG) } \\
\hline Before & $71.5 \pm 8.6$ & $71.5 \pm 10.5$ & 0.49 \\
\hline After & $71 \pm 7.3$ & $71.7 \pm 9$ & 0.34 \\
\hline$P$-value & 0.09 & 0.39 & \\
\hline \multicolumn{4}{|c|}{ BMI (kg/m2) } \\
\hline Before & $28.2 \pm 3.2$ & $27.4 \pm 3.7$ & 0.24 \\
\hline After & $27.9 \pm 2.9$ & $27.4 \pm 3.2$ & 0.55 \\
\hline$P$-value & 0.13 & 0.37 & \\
\hline \multicolumn{4}{|l|}{$\begin{array}{l}\text { Menstrual } \\
\text { cycle (day) }\end{array}$} \\
\hline Before & $47 \pm 8.99$ & $47.13 \pm 8.86$ & 0.33 \\
\hline After & $29.83 \pm 4.68$ & $47.11 \pm 8.72$ & 0.00 \\
\hline$P$-value & 0.004 & 0.34 & \\
\hline \multicolumn{4}{|l|}{ Hirsutism } \\
\hline \multicolumn{4}{|l|}{ mF-G score } \\
\hline Before & $12.8 \pm 3.4$ & $12.5 \pm 2.1$ & 0.78 \\
\hline After & $10.3 \pm 2.1$ & $12.7 \pm 5.6$ & 0.54 \\
\hline$P$-value & 0.34 & 0.76 & \\
\hline
\end{tabular}

Data expressed as mean $\pm \mathrm{SD}$ or median (range), $P$-values calculated by * Independent sample t-test or paired $\mathrm{T}$ test. BMI : body mass index.mFG score:modified Ferriman Gallway score. 
Table 3 shows that the comparison of hormonal profile between omega-3 group and control groups before and after treatment revealed no statistically significant difference between the 2 groups in the level of the studied hormones.

Table 3: Hormonal profile before and after treatment

\begin{tabular}{|c|c|c|c|}
\hline Hormone & Study group & $\begin{array}{l}\text { Control } \\
\text { group }\end{array}$ & $P$-value \\
\hline \multicolumn{4}{|l|}{ LH (IU) } \\
\hline Before & $9.4 \pm 5.3$ & $8.5 \pm 4.4$ & 0.52 \\
\hline After & $8.9 \pm 4.6$ & $8.1 \pm 3.4$ & 0.41 \\
\hline P-value & 0.23 & 0.12 & \\
\hline \multicolumn{4}{|l|}{ FSH (IU) } \\
\hline Before & $4.5 \pm 1.6$ & $4.4 \pm 2.0$ & 0.39 \\
\hline After & $4.3 \pm 1.3$ & $4.0 \pm 1.1$ & 0.25 \\
\hline$P$-value & 0.62 & 0.31 & \\
\hline \multicolumn{4}{|l|}{ LH/FSH ratio } \\
\hline Before & $2.18 \pm 1.15$ & $2.23 \pm 1.36$ & 0.79 \\
\hline After & $2.1 \pm 1.1$ & $1.9 \pm 0.9$ & 0.23 \\
\hline$P$-value & 0.32 & 0.09 & \\
\hline \multicolumn{4}{|l|}{$\begin{array}{l}\text { Free } \mathrm{T} \\
(\mathrm{pg} / \mathrm{ml})\end{array}$} \\
\hline Before & $1.5 \pm 0.67$ & $1.6 \pm 0.7$ & 0.47 \\
\hline After & $1.6 \pm 0.61$ & $1.4 \pm 0.68$ & 0.18 \\
\hline$P$-value & 0.13 & 0.12 & \\
\hline
\end{tabular}

Data expressed as mean $\pm \mathrm{SD}$, P-values calculated by paired $\mathrm{T}$ test or * Independent sample t-test. FSH: follicle stimulating hormone; LH: luteinizing hormone Free T: free testosterone

Table 4 shows the comparison of ovarian stromal and uterine artery Doppler between omega-3 group and control groups before and after treatment. Omega-3 polyunsaturated fatty acids induced a significant reduction of uterine arteries PI value $(P=.009)$ but there was no statistically significant change in ovarian stromal blood flow Doppler between the 2 groups $(P>0.05)$.
Table 4: Ultrasonic criteria before and after treatment

\begin{tabular}{lccc}
\hline $\begin{array}{c}\text { Doppler } \\
\text { value }\end{array}$ & Study group & $\begin{array}{c}\text { Control } \\
\text { group }\end{array}$ & P-value* \\
\hline Mean OA- RI & & & \\
before & $0.55 \pm .07$ & $0.56 \pm 0.07$ & 0.39 \\
after & $0.54 \pm .06$ & $0.55 \pm 0.07$ & 0.42 \\
$\begin{array}{l}\text { P value } \\
\text { Mean OA- PI }\end{array}$ & 0.31 & 0.33 & \\
before & $0.93 \pm 0.23$ & $0.93 \pm 0.21$ & 0.45 \\
after & $0.92 \pm 0.22$ & $0.96 \pm 0.22$ & 0.27 \\
$\begin{array}{l}\text { P value } \\
\text { Mean UA- PI }\end{array}$ & 0.45 & 0.58 & \\
before & $2.7 \pm 0.17$ & $2.5 \pm .017$ & 0.25 \\
after & $2.2 \pm 0.11$ & $2.5 \pm 037$ & 0.01 \\
$P$ value & $0.009 *$ & 0.14 & \\
\hline
\end{tabular}

Data expressed as mean $\pm \mathrm{SD}$, P-values calculated by paired T-test or *Independent sample t-test.

UA-PI: uterine artery pulsatility index;

OA-PI: ovarian stromal artery pulsatility index;

OA-RI: ovarian stromal artery resistance index.

\section{DISCUSSION}

In this prospective randomized study, 67 patients diagnosed by polycystic ovary syndrome were randomized to take $1000 \mathrm{mg}$ of omega-3 fatty acids supplementation daily or to receive no treatment for 3 months. Body weight, serum FSH, LH, free testosterone, uterine artery and ovarian stromal Doppler were compared before and after the study for both groups.

The dose of omega-3 supplementation that was used in this study $(1000 \mathrm{mg})$ was based on previously published studies by several authors ${ }^{[15-18]}$.

The results of this study showed that 3 months supplementation with omega-3 PUFAs in PCOS patients cased an improvement in menstrual cyclicity and uterine artery Doppler in comparison to control group. However, no statistically significant changes were found in weight, BMI, $\mathrm{mFG}$ score serum 
testosterone, LH, and FSH levels or ovarian stromal Doppler.

The current study showed that supplementation of omega-3 fatty acids significantly improved menstrual cycle regularity. These results were similar to previous published studies ${ }^{[12,15]}$. They showed that after 8-12 weeks supplementation with omega-3 PUFA, women with PCOS had significantly regular menstruation more than those who received placebo or no treatments.

The mechanism for the positive effect of omega-3 fatty acids on menstrual cyclicity is not clearly known, one suspected mechanism is that omega- 3 improves insulin sensitivity in PCOS women by increasing the anti-inflammatory mediators and reducing inflammation ${ }^{[9]}$.

Several clinical trials studied the effect of omega-3 on Insulin sensitivity showed that omega-3 increases insulin sensitivity as shown in the level of (HOMA) homeostatic model assessment of insulin resistance index and other insulin sensitivity markers.

A recently published systematic review on randomized trials comparing omega-3 supplementation with placebo or no treatment in the management of polycystic ovary syndrome ${ }^{[9]}$ found considerable variation. However, reviewers mentioned that omega-3 is effective in improving insulin resistance markers for those patients.

Other possible mechanism for regular menstruation is the decrease in testosterone concentration, testosterone level was significantly decreased after 8-week usage of omega-3 in comparison to placebo in some previous studies ${ }^{[13,16]}$, but the current study did not find change in testosterone, LH or FSH serum levels.

To our knowledge, this trial is the first randomized trial to study if omega-3 fatty acids has effects on uterine artery Doppler in PCOS patients. Previous study reported the effect of omega- 3 fatty acids on uterine artery in patients with repeated miscarriages due to impaired uterine perfusion showed that omega-3 fatty acids improves uterine artery blood flow and decrease its resistance ${ }^{[12]}$.

This study showed that omega-3 polyunsaturated fatty acids has positive effect on uterine artery but no effect on ovarian stromal blood flow. These result similar to study by (Lazzarin et al., 2009) ${ }^{[12]}$ who found that both aspirin and omega-3 polyunsaturated fatty acids lead to improved uterine artery blood flow in patients with repeated abortions due to defective uterine perfusion.

It seems that omega-3 fatty acids supplementation can provide a vasodilatatory effect by rearrangement of prostaglandins secretion. In particular, omega-3 may alter the prostacyclin/thromboxane ratio by decreasing thromboxane A2 synthesis at the platelet level ${ }^{[6]}$.

The current study did not find any effect of omega-3 PUFA on ovarian stromal blood flow in contrast to a study by (Kim et al., 2010) ${ }^{[11]}$ who found that both metformin and omega-3 PUFAs supplementation lead to decrease in ovarian stromal blood flow in PCOS patients.

This study did not show significant difference in weight before and after omega-3 supplementation in PCOS patients, this agrees with a metanalysis ${ }^{[18]}$ that was published recently which analyzed eight RCTs and reported the effect of omega-3 polyunsaturated fatty acids on BMI finding no strong evidence that the omega-3 fatty acid has a statistically significant effect on BMI.

The current study had some limitations as it did not measure serum fatty acids fractions, insulin and insulin sensitivity markers. Also, it did not assess luteal phase serum progesterone which support that reported bleeding resulted from ovulatory cycles, rather than only changes in menstrual flow. In addition, duration of the study was short.

\section{CONCLUSION}

In conclusion, our data suggested that omega-3 fatty acids supplementation may be a useful adjunct in the treatment to improve menstrual cyclicity and enhance uterine artery Doppler blood flow in patients with PCOS especially those who have defect in endometrial receptivity and subsequent infertility or repeated abortions because of high uterine artery impedance and resistance.

\section{CONFLICT OF INTEREST}

There are no conflicts of interest.

\section{REFERENCES}

1. Rodgers, R. J. et al. (2019) 'Is polycystic ovary syndrome a 20th Century phenomenon ?', Medical Hypotheses. Elsevier, 124(January), pp. 31-34.

2. Teede, H. J. et al. (2018) 'Recommendations from the international evidence-based guideline for the assessment and management of polycystic ovary 
syndrome',human reproduction update 33(9), pp. $1602-1618$.

3. Dokras, A. et al. (2018) 'Androgen Excess- Polycystic Ovary Syndrome Society: position statement on depression, anxiety, quality of life, and eating disorders in polycystic ovary syndrome. Fertility and Sterility ${ }^{\circledR}$ Vol. 109, No. 5, May 2018 ,American Society for Reproductive Medicine, Published by Elsevier Inc.

4. Dhingra, D. et al. (2017) 'Original article : Doppler flow velocities of uterine and ovarian arteries \& hormonal patterns in patients with Polycystic Ovary Syndrome ( PCOS )':, (July), pp. 48-57.

5. Brown, H. M. and Russell, D. L. (2014) 'Blood and lymphatic vasculature in the ovary: Development, function and disease', Human Reproduction Update, 20 (1), pp. 29-39.

6. Hu, X. et al. (2019) Effects of OMEGA-3 polyunsaturated fatty acids on steroidogenesis and cellular development in PCOS rats, Food Funct., 2019, 10, 2504-2514, The Royal Society of Chemistry.

7. Lands, B. (2017) 'Highly unsaturated fatty acids (HUFA) mediate and monitor food's impact on health', Prostaglandins and Other Lipid Mediators. Elsevier, (January), pp. 1-7

8. Calder, Philip C. 2015. "Marine OMEGA-3 Fatty Acids and Inflammatory Processes: Effects, Mechanisms and Clinical Relevance." Biochimica et Biophysica Acta (BBA) - Molecular and Cell Biology of Lipids 1851 (4). Elsevier B.V.: 469-84.
9. Candella, C. et al. (2015) 'The Role of OMEGA-3 Fatty Acids in Diets', Journal of the American College of Nutrition, 34(April), pp. 42-47.

10. Karakas, S. E. et al. (2016) 'Changes in plasma metabolites and glucose homeostasis during OMEGA-3 polyunsaturated fatty acid supplementation in patients with polycystic ovary syndrome', BBA Clinical. Elsevier B.V., 5, pp. 179-185.

11. Kim, C.-H. et al. (2010) 'Effect of Omega-3polyunsaturated fatty acids and metformin on ovarian morphology and intraovarian blood flow in patients with polycystic ovary syndrome', Fertility and Sterility. Elsevier Ltd, 94(4), p. S194.

12. Khani B, Mardanian F, Fesharaki SJ(2017). Omega-3 supplementation effects on polycystic ovary syndrome symptoms and metabolic syndrome. J Res Med Sci 2017;22:64.

13. Nasri, K. et al. (2017) 'The Effects of Omega-3 Fatty Acids Supplementation on Gene Expression Involved in the Insulin and Lipid Signaling Pathway in Patients with Polycystic Ovary Syndrome.', Hormone and metabolic research, 2017, 45 (4) pp.213-18

14. Phelan, N. et al. (2011) 'Hormonal and metabolic effects of polyunsaturated fatty acids in young patients with polycystic ovary syndrome: results from a cross-sectional analysis and a randomized placebocontrolled, crossover trial American Journal of Clinical Nutrition 2011;93:652-62. 